\title{
Three cases of spontaneous duodeno-colic fistula
}

\author{
BRUCE TORRANCE AND COLIN JONES \\ From the Department of Gastroenterology, Manchester Royal Infirmary
}

SUMMARY Three cases of duodeno-colic fistula are presented, where a communication was demonstrated between the anterior aspect of the third part of the duodenum and the transverse colon at the point of mesocolic attachment. At operation the fistulae were found to be some 6 to $9 \mathrm{~cm}$ in length, and histological examination showed a well marked muscular layer with an intact epithelium.

In view of the absence of any explanatory pathology, an embryological basis is suggested as the likeliest explanation for their origin.

All previously reported cases of duodeno-colic fistula have been associated with some other local pathological process (Aird, 1957). In the three cases presented, no such process could be demonstrated. The events which led to the discovery of the fistula are interesting and demonstrate how such a lesion can be misdiagnosed.

\section{Case Reports}

CASE

A 48-year-old woman was referred from another hospital. For two years she had suffered from severe watery diarrhoea. She had become extremely emaciated and lost over 3 stone in weight. Sigmoidoscopy revealed minor inflammatory changes in the rectum and a barium enema examination showed appearances consistent with a diagnosis of ulcerative colitis. Treatment with salazopyrin and predsol retention enemata had little effect on her symptoms. The original barium enema showed no evidence of a duodeno-colic fistula.

Because of her poor response to treatment she was referred to our department for panproctocolectomy. Further radiological investigations, however, including a barium meal and a barium enema (Fig. 1), demonstrated a fistulous connexion between the third part of the duodenum and the transverse colon.

An exploratory laparotomy was carried out. It was only after a careful search with full mobilization of the hepatic flexure and the duodenal loop that a fistulous track was found, lying between the layers of the transverse mesocolon (Fig. 2). The

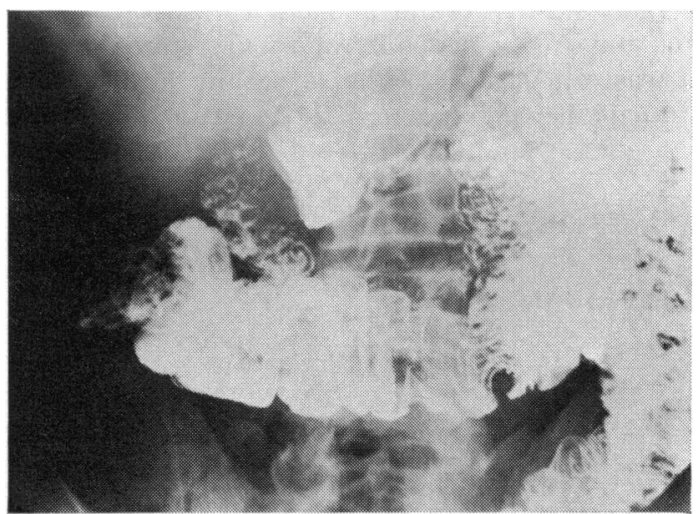

Fig. 1 Barium enema showing passage of barium into the duodenum and stomach from the proximal transverse colon.

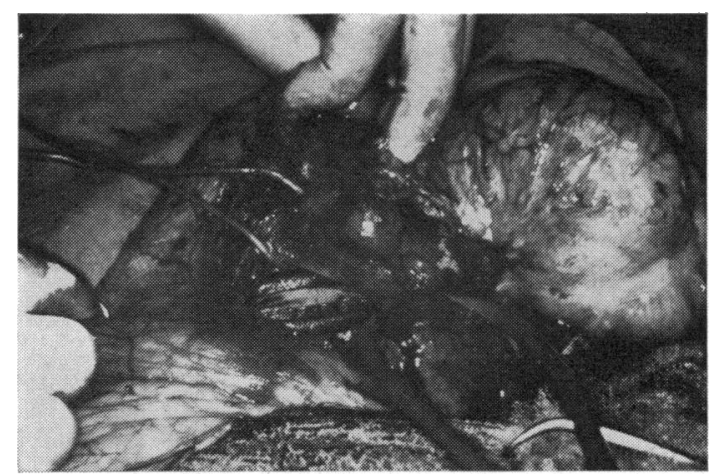

Fig. 2 Fistulous tract between the third part of the duodenum and the transverse colon (case 1). 
track was $9 \mathrm{~cm}$ long and $1 \mathrm{~cm}$ in width, extending from the anterior aspect of the third part of the duodenum, immediately to the right of the superior mesenteric vein to the proximal transverse colon. Several diverticula could be seen along the track itself, but none along the transverse colon or duodenum. No abnormality could be detected in the duodenum or transverse colon at the site of the connexion. The track itself was removed.

The patient made an uneventful recovery and rapidly regained her former body weight. When seen 18 months after her operation she was completely free from any abdominal symptoms.

\section{CASE 2}

A West Indian male aged 51 years presented with a 30-year history of intermittent diarrhoea. In 1945 he had been discharged from the Royal Air Force, and at that time a diagnosis of idiopathic steatorrhoea had been made.

Later he was found to have a macrocytic anaemia and was treated with folic acid and vitamin $B_{12}$. It was not until 1968 that a barium meal demonstrated the presence of a duodeno-colic fistula

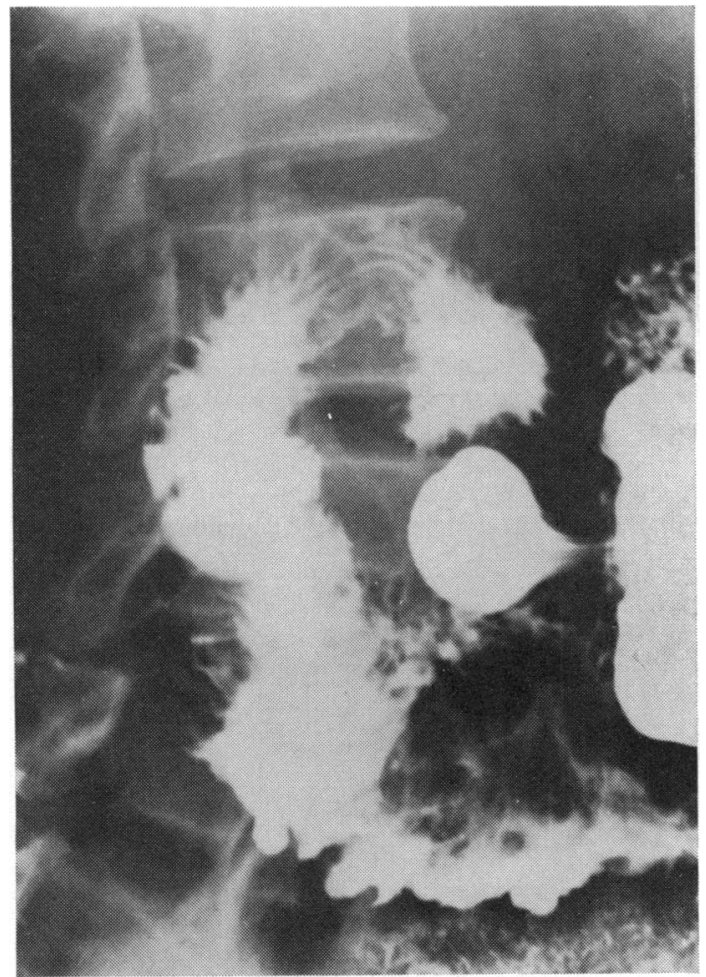

Fig. 3 Barium meal showing passage of barium into the transverse colon.
(Fig. 3). At that time he was found to excrete $64 \mathrm{~g}$ of fat over a three-day period.

At laparotomy, again not without a careful search, a duodeno-colic fistula was found. The track was $6 \mathrm{~cm}$ in length and $8 \mathrm{~mm}$ in width, connecting the third part of the duodenum to the transverse colon.

Following excision of the fistula, the patient's symptoms were entirely relieved. The faecal fat content returned to normal and within a year the blood picture was also normal. When seen three and a half years after operation he was entirely symptom free.

\section{CASE 3}

A 47-year-old woman gave a history of 10 years' intermittent diarrhoea. When she presented she was passing up to 10 steatorrhoea stools per day (faecal fat content over three days, $56 \mathrm{~g}$ ). Her history dated back to her last pregnancy.

Again, a barium enema demonstrated a duodenocolic fistula. At operation a fistulous track $7 \mathrm{~cm}$ in length and $1 \mathrm{~cm}$ in width was removed (Fig. 4). Seven years later she is still free from symptoms.

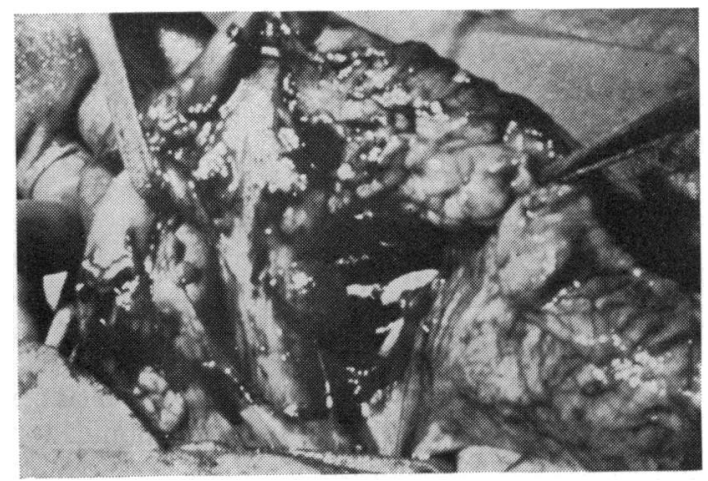

Fig. 4 Duodeno-colic fistula (case 3).

\section{Histology}

The findings were similar in all three cases. At one end of the track the mucous membrane was duodenal in type, and at the other, with a fairly sudden transition, the mucosa became typical of the large bowel. On all sections a well represented circular muscular coat (Fig. 5) was seen, except at the site of the diverticulae; here it was absent. The mucosal and muscular coats became continuous with those of the duodenum and transverse colon.

Apart from a minor infiltration with lymphocytes and plasma cells, no other pathological process could be demonstrated. 


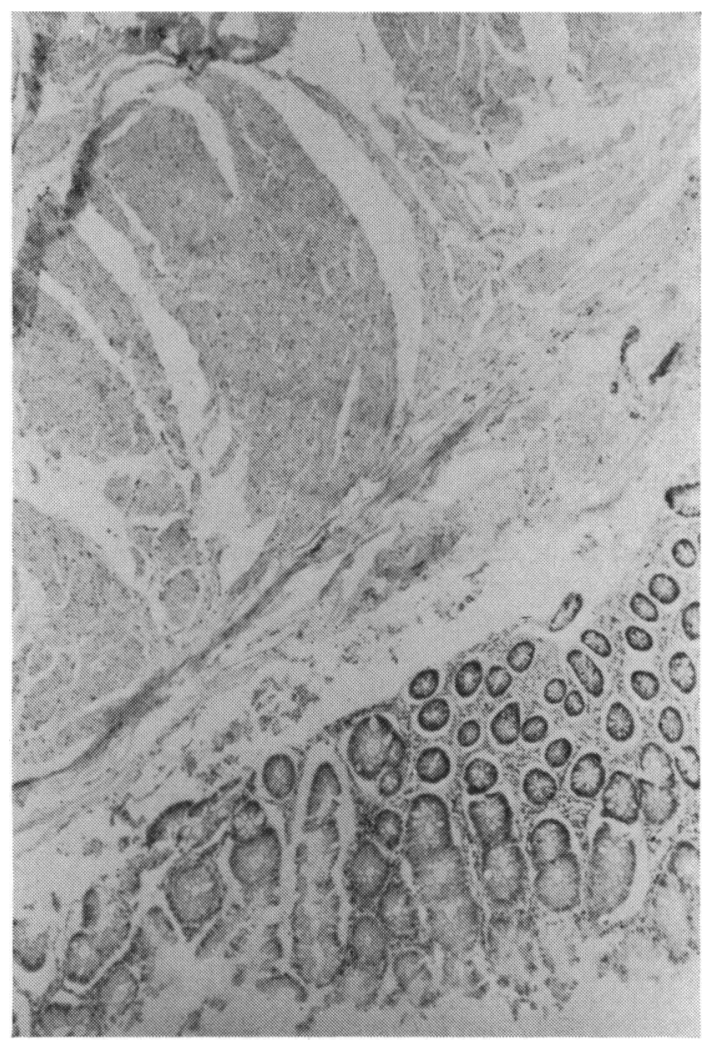

Fig. 5 Histological section of the tract at the midpoint where there is a transition of the mucous membrane from small bowel to large bowel type. Note the well marked muscular layer.

\section{Aetiology}

In the absence of any predisposing pathology and because of the well marked mucosal and, in particular, muscular coats, an embryological cause is suggested.

During the sixth week of development the embryo is at the $10 \mathrm{~mm}$ stage (Fig. 6). At this time the gut has outgrown the vertebral column and comes to form a U loop projecting out into the extraembryonic coelom. The developing duodenum and transverse colon come to lie close to one another. The approximation is enhanced by a twist in the $\mathrm{U}$ loop so that the distal limb crosses to the left of the proximal limb. At this time there is still multipotential mesenchymal tissue in the region and it is possible that some connexion is established (Ashley, 1970).

Later the gut becomes withdrawn into the coelomic cavity. By a process of extension and rotation,

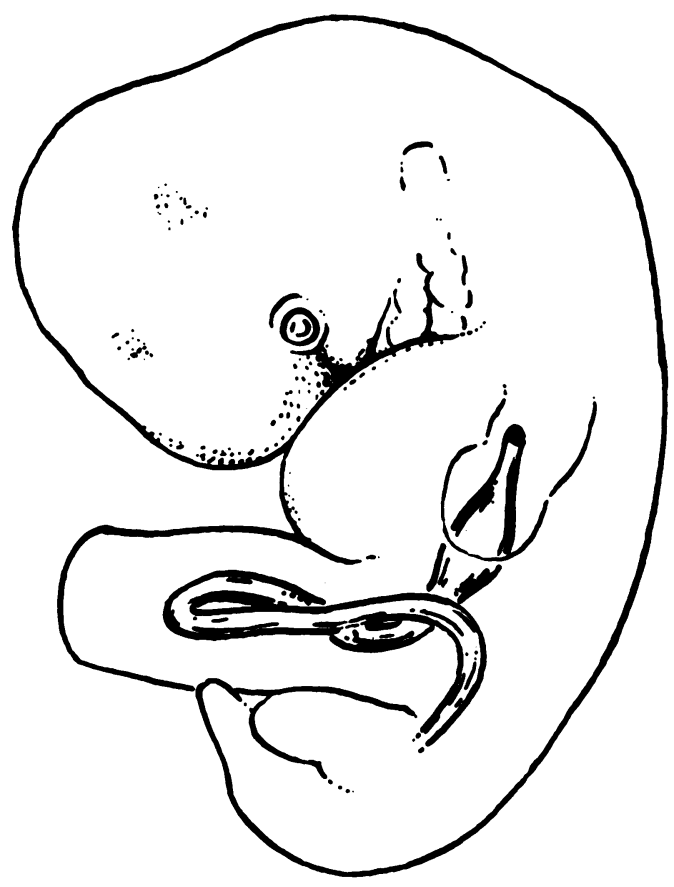

Fig. 6 Embryo at six weeks showing prolapse of the primitive gut into the extraembryonic coelom.

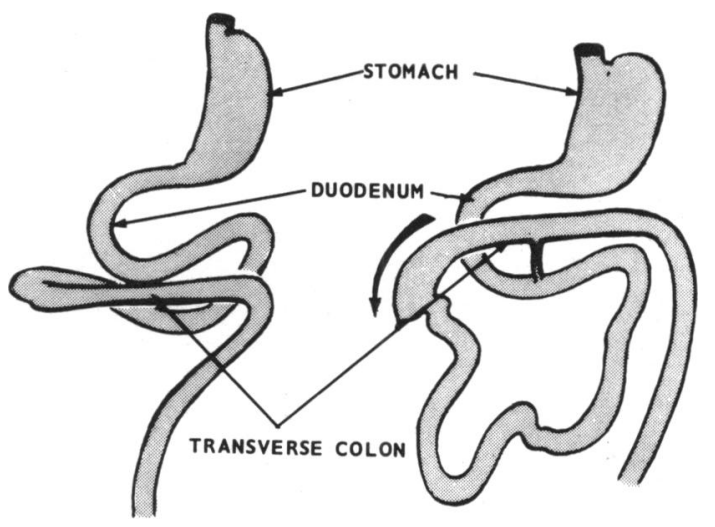

A

B

Fig. 7 Diagram to illustrate the relative movements of the developing small and large bowel, but with the development of a fistulous connexion in a $10 \mathrm{~mm}$ embryo. 
the caecum comes to lie on the right side of the abdominal cavity (Fig. 7). This relative movement of large bowel on small bowel could lead to an increase in the length of the fistulous connexion. Why this type of fistulous track should remain symptomatically quiescent until middle age is difficult to explain. Intestinal dysfunction will occur under two circumstances: (a) When the fistula transmits more than its fair share of duodenal contents. (b) When colonic bacteria invade the duodenum and small intestine.

It is conceivable that if peristaltic activity in the wall of the fistula proceeds in an aboral direction and only a limited quantity of duodenal contents is bypassed into the colon, this situation would be compatible with normal health. If, however, this protective effect of peristalsis became impaired, then retrograde colo-duodenal reflux would occur with colonization of the small intestine with bacteria. This theory might account for the fact that symptoms in our three cases only occurred later in life. The development of a diverticulum along the track, as happened in two out of the three cases, might have a similar effect.
It seems likely that this condition has been overlooked in the past, since during a laparotomy for the investigation of unexplained steatorrhoea or diarrhoea, few surgeons would proceed to the length of mobilizing the hepatic flexure and the duodenum and splitting the layers of the mesocolon. Unless this step is taken, the fistula will be missed. Indeed, in two of our three cases, despite the fact that we had radiological proof of a fistula, this was only revealed after considerable dissection. It is possible that, in some cases, a colectomy has been performed with a diagnosis of ulcerative colitis. During division of the transverse mesocolon with clamp and ligation, a surgeon would be unlikely to notice the presence of the fistula and the operation would, of course, be curative. We suspect that this condition may occur with sufficient frequency to merit consideration in any case of unexplained diarrhoea or steatorrhoea.

\section{References}

Aird, I. (1957). Companion in Surgical Studies, 2nd ed., p. 790. Livingstone, Edinburgh.

Ashley, T. (1970). Personal communication. 